\title{
Subgroups of Attention Deficit Disorder (ADHD). A Preliminary Clinical Descriptive Study
}

\author{
Castejón OJ*, Galindez P, Torres IA, Merchan F, Villasmil A, Diaz M, Casanova, L Grumbaum E and \\ Salones de Castejón M
}

Institute of Biological Research "Drs. Orlando Castejón and Haydee Viloria de Castejón "and Institute of Clinical Neurosciences, Castejón Foundation, Venezuela

*Corresponding author: Institute of Biological Research “Drs. Orlando Castejón and Haydee Viloria de Castejón "and Institute of Clinical Neurosciences, Castejón Foundation, Venezuela

ARTICLE INFO
Received: 崑 May 02, 2019
Published: 跷 May 09, 2019

Citation: Castejón OJ, Galindez P, Torres IA, Merchan F, Villasmil A, Diaz M, Casanova, L Grumbaum E, Salones de Castejón M. Subgroups of Attention Deficit Disorder (ADHD). A Preliminary Clinical Descriptive Study. Biomed J Sci \& Tech Res 17(5)-2019. BJSTR. MS.ID.003070.

Abbreviations: AD: Attention Deficit; ADHD: Attention Deficit Hyperactivity Disorder; BIP: Basic Information Processing; IBEF: Inhibitory-Based Executive Function; ASD: Autism Spectrum Disorders

\begin{abstract}
One hundred sixty (160) infant patients, ranging from 3 to 12 years-old, with hyperexcitability and attention deficit were examined from the psychological and clinical point of view. We have found fifth subtypes of neurodevelopmental subtypes in the study of ADHD. One first subtype with attention deficit alone (AD). A second combined subtype with hyperexcitability and attention deficit (ADHD), a third subgroup with co-occurrence of ADHD and autism spectrum disorder (ADHD-AED), a four subgroup with hyperexcitability and attention deficit (ADHD) and aggressivity (ADHD-A) and a fifth subgroup ADHD and seizures (ADHD-S). These groups exhibited at least some of the following associated risk factors and comorbidities: perinatal hypoxia, prematurity and low weight at birth, behavioral abnormities, oppositional defiant disorder, anxiety, auto- and hetero-aggressivity, autism spectrum disorder, language, learning and sensorineural hearing loss, anorexia or hyperphagia, mental retardation, social isolation, dyslexia, dysgraphia, cognitive deficit, sleeping disorders, and suicidal thoughts, planning and attempts. Some non-nervous system comorbidities, such as pulmonary diseases and allergic reactions. Locomotor abnormalities as genus valgo and flat feet also were frequently found. The following maternal risk factors were detected: stress, placenta praevia, conjugal conflicts, diabetes, high blood pressure, anxiety and depression and critical poverty.
\end{abstract}

Keywords: Hyperexcitability; Attention Deficit; Clinical Study; Comorbidities

\section{Introduction}

Attention deficit/hyperactivity disorder (ADHD) is a term used to describe a constellation of inappropriate levels of inattention and impulsivity. The history of name changes from minimal brain syndrome, to hyperkinetic syndrome, to what is now known as ADHD, reflects the influence of neurology, pediatrics, and psychiatry. This "evolutionary" process has been replete with controversy stemming from the diverse views of broad disciplines that have attempted to define its range, scope, and treatment [1]. According to Sell-Salazar [2]. There are two specific syndromes:

a) Attention deficit disorder, without hyperactivity;

b) A more complex syndrome, with hyperactivity, and traits of impulsivity, accompanied by attention deficit disorder.
The etiology of this syndrome is still unclear, but there has been some hypothesis about hypo perfused areas in the frontal lobe, in some patients where PET has been available. There has been also some response to stimulants; which leads to the conclusion that there is some relation with neurotransmitters, like noradrenaline and dopamine, as a determinant factor in the origin of this process.

The associated comorbidity most frequently associated with ADHD are: Tourette syndrome, generalized development disorders, learning disorders, coordination development disorders, behavioral disorders, anxiety disorders, affective disorders and mental retardation. Montiel Nava [3] reported a clinical presentation of attention deficit/hyperactivity disorder as a function of the gender. Attention-deficit/hyperactivity disorder 
(ADHD) is a neurobiological condition of childhood onset with the hallmarks of inattention, impulsivity, and hyperactivity. Inattention includes excessive daydreaming, disorganization, and being easily distracted. Impulsivity manifests as taking an action before fully thinking of the consequences. Hyperactivity includes an excessive rate of speech and motor activity. Complications of ADHD include academic failure due to deficit of learning and memory and neurosensory alterations, low self-esteem, poor work performance, substance abuse, criminal justice issues, and social problems. ADHD is predominately due to decreased activity in the frontal lobe $[4,5]$. Risk factors include familial stressors, anxiety disorders, learning disabilities, abnormal brain development, heritability, and dopamine polymorphisms [6]

ADHD is predominately due to a decreased activity in the frontal lobe. and deficits in executive function (EF). There is a common dysfunction in the processing of click and speech stimuli at the brainstem level in children with suspected ADHD [7]. There is a putative ADHD-related deficit in basic information processing (BIP) and inhibitory-based executive function (IB-EF) in individuals in the subclinical and full clinical ranges of ADHD [8]. Autism spectrum disorders (ASD) and attention deficit hyperactivity disorder (ADHD) are two of the most common neurodevelopmental disorders, with a high degree of co-occurrence [9] (Johnson et al., 2014). Studies have shown that ADHD is characterized by multiple functional and structural neural network abnormalities including most prominently fronto-striatal, but also fronto-parietotemporal, fronto-cerebellar and even fronto-limbic networks $[10,11]$. Evidence from longitudinal structural imaging studies has shown that ADHD is characterized by a delay in structural brain maturation [12]. In the present paper we describe in a preliminary way a clinical and cualitative study of children with ADHD. We characterize the homogenous subgroups, subtyping patients according to clinical course, symptom dimensions, comorbidity patterns and neurocognitive and neurophysiological dysfunctions in order to attend to a precision medicine and a rational therapy.

\section{Material and Methods}

One hundred sixty (160) infant patients, ranging from 3 to 12 years-old, with hyperexcitability and attention deficit were clinically studied at the Clinical Neuroscience Institute Outpatient Clinic at the Clinical Home San Rafael de Maracaibo. They were previously examined from the psychological point of view at the Departments of Psychology of CETRO, San Rafael Home Clinic, and at different Public and private Psychology Centers of Maracaibo City. This study was carried out following some DMS V guidelines. The ethical principles of Helsinki Declaration for clinical research in human beings have been applied in the present study. Parent consent was obtained in each case.

\section{Results}

We have found fifth subtypes of neurodevelopmental disorders in the study of ADHD. One first sub type with attention deficit alone
(AD) (3\%). A second combined subtype with hyperexcitability and attention deficit (ADHD) (59\%), a third subgroup with cooccurrence of ADHD and autism spectrum disorder (ADHD-AED) (12\%), a four group with ADHD and aggressive behavior (ADHD-A) (22\%), and a fifth subgroup with seizures (ADHD-S) (4\%). The first subgroup (AD) showed perinatal hypoxia, psychomotor, cognitive deficit and language disorders. The second subgroup (ADHD), the infant patients exhibited some of the following associated comorbidities: perinatal hypoxia, prematurity and low weight at birth, behavioral abnormities, oppositional defiant disorder, anxiety, auto- and hetero aggressivity, autism spectrum disorder, language disorders, learning deficit and sensorineural hearing loss, strabismus, anorexia or hyperphagia, and mental retardation. Social isolation, dyslexia, dysgraphia, lepto-scripture difficulties, cognitive deficit, sleeping disorders, talking during sleep, suicidal thoughts, planning and attempts, aggression post-Chikungunya and Cytomegalovirus infection, parenteral abuse of child.

Some non-nervous system comorbidities, such as pulmonary diseases, allergic reactions, locomotor abnormalities as genus valgo and flat feet also were frequently found. The following maternal risk factors were detected: labor stress, placenta praevia, conjugal conflicts, diabetes, critical poverty, high blood pressure, and anxiety and depression. The third subgroup with co-occurrence of ADHD and autism spectrum disorder (ADHD-AED) displayed repetitive and stereotyped patterns of behavior, and, fear and social isolation. The four group with aggressivity (ADHD-A) showed impulsivity, school conductual problems as cardinal dimensions of externalizing behavior problems, poorer school results and social adaptation, lower social competency, poorer communication, and/or diminished adaptive skills. Some patient exhibited signs of physical and psychological child abuse at home. The fifth subgroup ADHD and seizure disorders (ADHD-Z) showed generalized tonic-clonic epilepsy and partial convulsions or type petit mal or absences.

\section{Discussion}

\section{The Subgroup Types of ADHD}

In the present paper we have distinguished fifth subtypes of neurodevelopmental disorders in the study of ADHD. Park [13] compared two subtypes, the attention-deficit/ hyperactivity disorder (ADHD) combined subtype (ADHD-C) to the ADHD inattentive subtype (ADHD-I) in terms of genetic, perinatal, and developmental risk factors, as well as clinical and neuropsychological characteristics. According to these Authors the inattentive subtype of ADHD is different from the combined subtype in many parameters including severity of symptoms, comorbidity, neuropsychological characteristics, and environmental risk factors.

\section{Brain Microcircuit Alteration in ADHD}

As we have dreported in a previous publication [14,15] structural neural network abnormalities include fronto-parietotemporal, fronto-cerebellar and even fronto-limbic networks. The dopaminergic and noradrenergic system systems have been 
postulated as involved in the pathophysiology of ADHD, such as cognitive processes, such as arousal, working memory, and response inhibition, all of which are typically affected in ADHD [16] Patients showed significantly reduced dorsal caudate functional connectivity with the superior and middle prefrontal cortices as well as reduced dorsal putamen connectivity with the Para hippocampal cortex. [17]. Akutagava Martins [18] postulate a role for glutamate in ADHD in the glutamatergic genes associated with cognitive and clinical characteristics of ADHD individuals.

\section{Cerebellar Involvement}

Stoodley [19] results suggest that different cerebellar regions are affected in in autism spectrum disorder (ASD), ADHD, and dyslexia, and that these cerebellar regions participate in functional networks that are consistent with the characteristics symptoms of each disorder. According to Wolafnczyk and Picazio and Koch $[20,21]$ there is a causal role of the effective cerebello-cortical connectivity in motor inhibition. Understanding the neurophysiological mechanisms that mediate motor inhibition through the cerebellum could be essential to design new rehabilitative protocols for treating several neurological and psychiatric disorders characterized by disinhibited behavior, such as addiction, schizophrenia, attention deficit hyperactivity disorder (ADHD) and Parkinson's disease. In relationship with the involvement of cerebellum in aggression and ADHD, the cerebellar regions showed functional connectivity with frontoparietal default mode, somatomotor, and limbic networks. In ADHD, autism spectrum disorder (ASD) the clusters were part of dorsal and ventral attention networks, and in dyslexia the clusters involved ventral attention, fronto-parietal, and default mode networks. The results suggest that different cerebellar regions are affected in sensory processing abilities, ASD, ADHD, and dyslexia, and these cerebellar regions participate in functional networks that are consistent with the characteristic symptoms of each disorder [22].

\section{Sensory Processing Impairments}

Sensory processing impairments, mainly strabismus and hearing deficit, were frequently observed. Children with ADHD may present sensory processing impairments, which may contribute to the inappropriate behavioral and learning responses displayed by children with ADHD. It also suggests the importance of understanding the sensory processing difficulties and its possible contribution to the ADHD symptomatology. Complications of ADHD include academic failure, low self-esteem, poor work performance, substance abuse, criminal justice issues, and social problems. Shimizu [22] also reported that children with ADHD may present sensory processing impairments, which may contribute to the inappropriate behavioral and learning responses displayed by children with ADHD. It also suggests the importance of understanding the sensory processing difficulties and its possible contribution to ADHD symptomatology. Maternal stress and pathology during pregnancy, perinatal hypoxia, risk factors involved, comorbidities, endophenotypes and ADHD, child language impairment, learning and memory deficit, physical, psychological and sexual child abuse, eating disorders, aggressive behavior and ADHD, learning and memory deficit were examined and described in detail in a previous publication [15].

\section{Separation Anxiety Disorders and ADHD}

We have above described ADHD children with fear and nervousness and mood disorders. These children often reject the clinical examination. Separation anxiety was observed in those children whose parents have migrated from their home country searching for a better quality of life or by being separated as conjugal partners. According to Christopher [23], ADHD children have more active frontal, temporal, parietal, and occipital lobes during concentration than children diagnosed with anxiety in the same brain areas.

\section{Co-Occurrence of ADHD and Autism Spectrum Disorder (ASD)}

In the present study we have reported a subgroup characterized by co-occurrence of ADHD and Autism spectrum disorder (ASD-A). According to Van Steijn [24], autism spectrum disorder (ASD) and attention-deficit/hyperactivity disorder (ADHD) share about 50$72 \%$ of their genetic factors, which is the most likely explanation for their frequent co-occurrence within the same patient or family. ASD dimension (reflecting restricted, repetitive and stereotyped patterns of behavior, interests and activities) showed the strongest association with dimensions of ADHD, on a phenotypic, genetic and environmental level [25]. In the present study we found patients with autism spectrum disorders (ASD) and attention deficit hyperactivity disorder (ADHD), which are two of the most common neurodevelopmental disorders, with a high degree of cooccurrence [9].

\section{The Need for Deconstructing Current Diagnostic Groups and Precision Medicine}

According to Pallanti and Grassi (2014) there is a critical need for deconstructing current diagnostic groups with biomarkers to predict and improve response to treatment. Current available treatments are still syndrome-based rather than network dysfunctions-based. Identifying the homogenous subgroup, subtyping patients according to comorbidity patterns, symptom dimensions, clinical course, neurocognitive and neurophysiological dysfunctions, which could represent an essential first step in the direction of a 'precision medicine' approach. The treatment of ADHD children will be the subject of a future publication.

\section{Acknowledgement}

This investigation has been supported by Biological Research Institute "Drs. Orlando Castejón and Haydee Viloria Castejón". Faculty of Medicine, Zulia University and Clinical Neuroscience Institute. Castejón Fundation. San Rafael Clinical Home at Maracaibo, Venezuela. 


\section{References}

1. Sangare J (2000) ADHD: making the appropriate pediatric assessment Lippincotts Prim Care Pract 4(2): 193-206.

2. Sell Salazar F (2003) Attention deficit hyperactivity syndrome. Rev Neurol 37(4): 353-358.

3. Montiel Nava C, Montiel Barbero I, Peña JA (2007) Clinical presentation of attention deficit/hyperactivity disorder as a function of the gender. Invest Clin 48(4): 459-468.

4. Bokor G, Anderson PD (2014) Attention-Deficit/Hyperactivity Disorder. J Pharm Pract 27(4): 336-349.

5. Kulkarni M (2014) Attention Deficit Hyperactivity Disorder. Indian J Pediatr Transl Psychiatry 4: 435-439.

6. Hong SB, Zalesky A, Park S, Yang YH, Park MH, et al. (2015) COMT genotype affects brain white matter pathways in attention-deficit/ hyperactivitydisorder. Hum. Brain Mapp 36(1): 367-377.

7. Jafari Z, Malayeri S, Rostami R (2015) Subcortical encoding of speech cues in children with attention deficit hyperactivity disorder. Clin Neurophysiol 126(2): 325-332.

8. Salum GA, Sonuga Barke E, Sergeant J, Vandekerckhove J, Gadelha A, et al. (2014) Mechanisms underpinning inattention and hyperactivity: neurocognitive support for ADHD dimensionality. Psychol Med 44(15): 3189-3201.

9. Johnson MH, Gliga T, Jones E, Charman T (2015) Annual Research Review: Infant development, autism, and ADHD - early pathways to emerging disorders. J Child Psychol Psychiatry 56(3): 228-247.

10. Castejón OJ (2018a) Neural correlates of cortico-thalamic-stratiated and limbic pathway in ADHD. (Abstract). Venezuelan J Neurosci 1: 120.

11. Castejon OJ, Galindez P, Torres IA, Leal J, Villasmil A, et al. (2018b). Maternal Stress, Pregnancy Diseases and Child Hyperactivity and Attention Deficit. Biomedical Journal of Scientific \& Technical Research (BJSTR)12(2): 1-9.

12. Rubia K, Alegria AA, Brinson H (2014) Brain abnormalities in attentiondeficit hyperactivity disorder: a review. Rev Neurol 58 (Suppl1): 3-16

13. Park S, Cho SC, Kim JW, Shin MS, Yoo HJ, et al. (2014) Differential perinatal risk factors in children with attention-deficit/hyperactivity disorder by subtype. Psychiatry Res 219(3): 609-616.

\section{ISSN: 2574-1241}

DOI: 10.26717/BJSTR.2019.17.003070

Orlando J Castejón. Biomed J Sci \& Tech Res

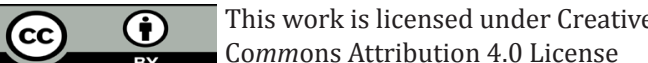

Submission Link: https://biomedres.us/submit-manuscript.php
14. Castejon OJ, Galindez P, Torres IA, Leal J, Villasmil A, et al. (2018b). Maternal Stress, Pregnancy Diseases and Child Hyperactivity and Attention Deficit. Biomedical Journal of Scientific \& Technical Research (BJSTR)12(2): 1-9.

15. Metin B, Krebs RM, Wiersema JR, Verguts T, Gasthuys R, et al. (2015) Dysfunctional modulation of default mode network activity in attentiondeficit/hyperactivity disorder. J Abnorm Psychol 124(1): 208-214.

16. Hong SB, Zalesky A, Park S, Yang YH, Park MH, et al. (2015) COMT genotype affects brain white matter pathways in attention-deficit/ hyperactivitydisorder. Hum Brain Mapp 36(1): 367-377.

17. Akutagava Martins GC, Salatino Oliveira A, Genro JP, Contini V, Polanczyk G, et al. (2014) Glutamatergic copy number variants and their role in attention-deficit/hyperactivitydisorder. Am J Med Genet B Neuropsychiatr Genet 165B(6): 502-509.

18. Stoodley CJ (2014) Distinct regions of the cerebellum show gray matter decreases in autism, ADHD, and developmental dyslexia. Front Syst Neurosci 8: 92.

19. Wolafnczyk T, Wolafnczyk T, Kolakowski A, Pisula A, Liwska M, et al. (2014) State and trait anxiety level and increase of depression among mothers of children with attention deficit hyperactivity disorder and conduct disorder. pilot study Psychiatr Pol 48(4): 645-652.

20. Picazio S, Koch G (2014) Is Motor Inhibition Mediated by Cerebellocortical Interactions? Cerebellum 14 (1): 47-49.

21. Shimizu VT, Bueno OF, Miranda MC (2014) Sensory processing abilities of children with ADHD. Braz J Phys Ther 18(4): 343-352.

22. Christopher S. Martin, Mitch Earleywine, Timothy C Blackson, et al (1994) Aggressivity, inattention, hyperactivity, and impulsivity in boys at high and low risk for substance abuse. J Abn Child Psychol 22(2): 177203.

23. Van Steijn DJ, Richards JS, Oerlemans AM, de Ruiter SW, van Aken MA, et al. (2012) The co-occurrence of autism spectrum disorder and attentiondeficit/hyperactivitydisorder symptoms in parents of children with ASD or ASD with ADHD. J Child Psychol Psychiatry 53(9): 954-963.

24. Polderman TJ, Hoekstra RA, Posthuma D, Larsson H (2014) The cooccurrence of autistic and ADHD dimensions in adults: an etiological study in 17,770 twins. Transl Psychiatry 4: 435.

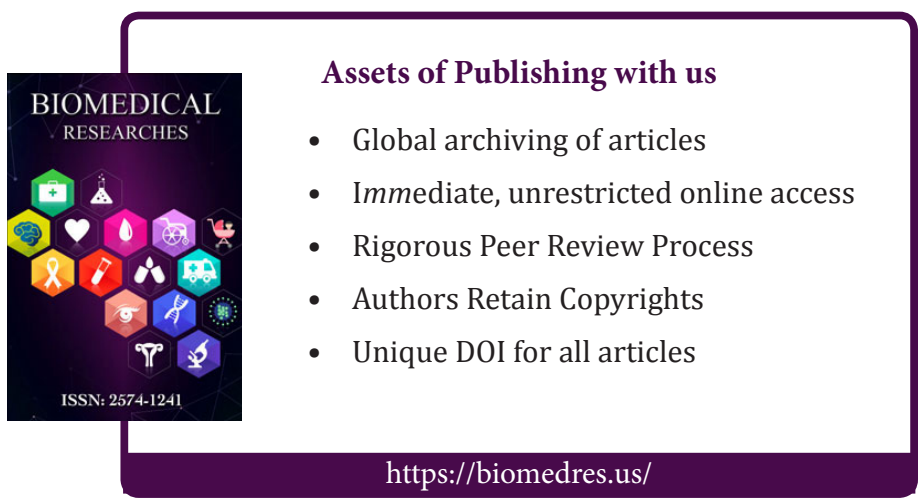

